Review article

\title{
Linguistic and interactional aspects that characterize consultations about medically unexplained symptoms: A systematic review
}

\author{
Inge Stortenbeker ${ }^{\mathrm{a}, *}$, WykeStommel ${ }^{\mathrm{a}}$, Sandra van Dulmen ${ }^{\mathrm{b}, \mathrm{c}, \mathrm{d}}$, Peter Lucassen ${ }^{\mathrm{b}}$, Enny Das ${ }^{\mathrm{a}}$, \\ Tim olde Hartman ${ }^{b}$ \\ a Centre for Language Studies, Radboud University, Nijmegen, The Netherlands \\ ${ }^{\mathrm{b}}$ Radboud University Medical Center, Radboud Institute for Health Sciences, Department of Primary and Community Care, Nijmegen, The Netherlands \\ ${ }^{\mathrm{c}}$ NIVEL (Netherlands Institute for Health Services Research), Utrecht, The Netherlands \\ ${ }^{\mathrm{d}}$ Faculty of Health and Social Sciences, University of South-Eastern Norway, Drammen, Norway
}

\section{A B S T R A C T}

Objective: The apparent absence of any specific underlying diseases challenges patient-provider communication about medically unexplained symptoms (MUS). Previous research focused on general communication patterns in these interactions; however, an overview of more detailed interactional and linguistic aspects is lacking. This review aims to gain a detailed understanding of communicative challenges in MUS consultations by synthesizing evidence from conversation and discourse analytic research.

Methods: A systematic review of publications using eight databases (PubMed, Embase, CINAHL, PsychINFO, Web of Science, MLA International Bibliography, LLBA and Communication Abstracts). Search terms included 'MUS', 'linguistics' and 'communication'. Additional studies were identified by contacting experts and searching bibliographies. We included linguistic and/or interactional analyses of natural patient-provider interactions about MUS. Two authors independently extracted the data, and quality appraisal was based on internal and external validity.

Results: We identified 18 publications that met the inclusion criteria. The linguistic and interactional features of MUS consultations pertained to three dimensions: 1) symptom recognition, 2) double trouble potential (i.e. patients and providers may have differing views on symptoms and differing knowledge domains), and 3) negotiation and persuasion (in terms of acceptable explanations and subsequent psychological treatment). We describe the recurrent linguistic and interactional features of these interactions.

Conclusions: Despite the presence of a double trouble potential in MUS consultations, validation of symptoms and subtle persuasive conduct may facilitate agreement on illness models and subsequent (psychological) treatment.

\section{Introduction}

The apparent absence of a specific underlying disease challenges patient-provider communication about medically unexplained symptoms (MUS). Whereas patients feel that their symptoms have biomedical causes, healthcare providers tend to highlight the importance of the psychosocial context in which symptoms have emerged [1]. This incongruence can make patients and healthcare providers feel powerless and frustrated $[2,3]$. Healthcare providers often feel unable to provide the right support [4] and no single effective treatment strategy exists for these patients [5], who account for $3-20 \%$ of all medical consultations $[6,7]$. This leaves the clinical encounter, and thus the communication between healthcare providers and patients, as a major site for symptom management [8]. Interactional and linguistic research can reveal im- portant insights in communication patterns by studying the actions that are performed, their design (e.g. grammar, pitch or intonation) and what happens next (i.e. the sequentiality) [9]. By gaining a more detailed understanding of linguistic (e.g. word choice) and interactional (e.g. turn-by-turn sequence design) aspects of communication, this type of research can facilitate improvement in clinical practice and policy [e.g. [10]].

Previous research has documented several relevant communication patterns in the MUS context, but an overview of interactional and discourse analytical research is still lacking. A systematic review of how linguistic and interactional aspects characterize natural patient-provider interactions about MUS can reveal important insights into communication patterns in various medical settings and provide a deeper understanding of such patterns $[11,12]$.

\footnotetext{
* Corresponding author at: Erasmusplein 1, 6525 HT Nijmegen, The Netherlands.

E-mail addresses: i.stortenbeker@let.ru.nl (I. Stortenbeker); w.stommel@let.ru.nl (W. Stommel); s.vandulmen@nivel.nl (S. van Dulmen); peter.lucassen@radboudumc.nl (P. Lucassen); h.das@let.ru.nl (E. Das); tim.oldehartman@radboudumc.nl (T. olde Hartman)
} 


\section{Methods}

We performed a synthesis of results according to the principles of meta-ethnography [13]. The review follows PRISMA guidelines [14]. ${ }^{1}$

\subsection{Literature search}

We searched for relevant publications in eight databases that include research in medicine, communication and linguistics (PubMed, Embase, CINAHL, PsychINFO, Web of Science, MLA International Bibliography, LLBA and Communication Abstracts) in April 2019. The search string combined search queries related to medically unexplained symptoms, communication and linguistics. For medically unexplained symptoms, we combined search terms used by Olde Hartman et al. [15], Hoedeman et al. [16] and terms used in the Dutch multidisciplinary guideline for MUS and somatoform disorders $[17,18]$. We based our search string for communication on a review by Mistiaen et al. [19] and the search string for linguistics on a review by Parry \& Land [20]. The full search strategy is shown in Appendix 1. We searched for additional studies by screening the reference lists in the included studies and other work by the included authors, by consulting experts on MUS or language and interaction, and by screening handbooks on health communication.

\subsubsection{Inclusion and exclusion}

We only included observational studies that analysed video and audio recordings of natural patient-provider interactions dealing with MUS. We adopted a broad definition of MUS as an umbrella term for various 'unexplained' symptoms, since we aimed to provide an overview of all relevant research conducted in this area. This included single-symptom MUS (e.g. tension headache or persistent dizziness), functional syndromes (e.g. fibromyalgia or irritable bowel syndrome) and undifferentiated somatoform/somatic symptom disorders $[15,17,18]$. All healthcare providers operating in medical or paramedical settings were eligible for inclusion. We considered studies to be linguistic or interactional when the methodology encompassed such analysis (e.g. conversation, narrative or discourse analysis) or when the study described linguistic categories, actions and/or prosodic or paralinguistic phenomena. Conversation analysis (CA) is a data-driven, ethno-methodological approach to studying 'sequences' in natural interactions, which aims to reveal structural patterns in spoken interactions [21,22]. Narrative analysis in medical settings focuses on how patients construct their illness stories [23], and discourse analysis encompasses a variety of research methods focusing on "language, meaning and context" [24]. Studies coding content features only without consideration of linguistic features were excluded [e.g. 25-27]. Qualitative and quantitative research methods were considered. All inclusion criteria are described in Table 1.

\subsubsection{Study selection}

Two reviewers (IS and either NH [medical student] or IP [PhD student]) independently screened the titles and abstracts of all the citations produced by the database search. They met up to discuss the findings. The reviewers independently read full the publications that seemed to match the inclusion criteria and subsequently discussed these publications. Disagreements were resolved through discussion and by consulting a third reviewer (PL). After selecting the relevant studies, we contacted experts and screened the reference lists of the selected publications to search for additional relevant publications. These studies were also discussed by two reviewers (IS and IP).
Table 1

Eligibility criteria for study selection.

Inclusion

\begin{tabular}{ll}
\hline & Inclusion \\
\hline \multicolumn{2}{l}{ Study eligibility criteria } \\
Participants & Patients with MUS in (para)medical settings \\
Data & Video and audio recordings of natural patient-provider interactions \\
Outcomes & Linguistic and interactional aspects \\
Design & Observational studies \\
Report eligibility criteria \\
Language & All languages (English abstract) \\
Year & Start database - April 2019 \\
Publication & Published studies or accepted for publication, book chapters, \\
status & dissertations, case reports \\
\hline
\end{tabular}

\subsection{Data extraction and synthesis}

The data were synthesized in an iterative process in which the interdisciplinary review team of CA experts, communication researchers and clinicians collaborated closely. We aimed to compare individual findings and create potential new interpretations. IS, ToH and PL screened all the included studies and established for each communicative practice what was addressed by whom and - if described - when, how and why. The data from each study were synthesized by at least two team members. All identified communicative practices in the individual studies were compared and grouped in an iterative process. We identified candidate dimensions by comparing the concepts and practices in one paper with practices assessed in others. We compared studies based on addressing specific practices (e.g. 'category-constrained questions' [28] and restricted question answer sequences [29]) or their communicative implications (e.g. dramatizing expressions to emphasize involuntary nature [30] and historical present to recreate a vivid experience [31]). Candidate dimensions were further refined in ongoing interdisciplinary team discussions, and by going back and forth to the original studies. This process continued during the writing of the review. Eventually, the team agreed upon three main dimensions covering most of the communicative practices addressed in the papers. To make sure no potentially relevant details were missed, IS read all the papers once more to further refine the analysis wherever possible.

\subsection{Quality appraisal}

This research additionally aimed to gain an understanding of the strengths and weaknesses of the methodological and analytical aspects of the included studies. Parry and Land [20] propose that, since it is not possible to perform a single assessment of quality for interactional research, two separate dimensions should be assessed: 1) the type and amount of data; and 2) the detail and depth of analysis. We compared these criteria to other reporting criteria for qualitative research $[32,33]$ and a checklist for reporting CA research [34]. Ongoing team discussion generated a quality appraisal form based on criteria given by Parry and Land [20], supplemented with additional items describing CA principles and participant characteristics [34]. The form (see Appendix 2) allowed us to gain an understanding of the data characteristics and the depth and detail of analysis. The description of data characteristics, representing the external validity, included items such as 'number of interactions', 'patient characteristics' and 'provider characteristics'. The depth and detail of the analysis, representing the internal validity, included items such as "Are established analytic findings used as 'tools' in the analysis?" and "Does analysis include examination of the sequential environment in which practices occurs?". Four included studies were scored by IS and WS. IS continued the quality appraisal for the remaining studies since no major interpretation differences

\footnotetext{
1 The study protocol was registered in PROSPERO (CRD42018095405).
} 
were observed between the scorers. Doubts about the quality appraisal were resolved in discussion with WS.

\section{Results}

The database search yielded 5367 publications after removing duplicates. Of these, 108 records seemed to meet the inclusion criteria based on the title and abstract. After assessing the full publications, 12 articles were selected for inclusion. Important reasons for exclusion were that the studies did not describe natural patient-provider interactions ( $n=58)$ or any linguistic or interactional aspect $(n=17)$. We identified only one quantitative study [35], which was excluded from further analysis since its methodology differed too much from the qualitative studies. Our additional search yielded six additional publications. One publication retrieved by screening publications from the included authors also appeared in the database search but had been missed by both reviewers [36]. Another study, retrieved by screening reference lists, did not appear in the database search (no full publication available; search strategy corresponded with information provided in full text) [29]. Other studies were book chapters $(n=3)$ [37-39], and a chapter from an unpublished $\mathrm{PhD}$ dissertation [30]. This resulted in a total of 18 included publications (Fig. 1), based on 14 different studies.

Eight publications were based on neurology settings, five on general practice, two on chronic fatigue syndrome clinics, two on physiotherapy and one study involved internal medicine (Table 2). Most of the studies were performed in the United Kingdom. Five publications were based on the same dataset about functional neurological symptoms $[37,38,40-42]$. Most of the studies analysed consultations with patients suffering from severe MUS, e.g. based in specialized secondary care units $[29,43]$ or neurology centres $[28,37,38,40-42,44,45]$, presenting at least four-six symptoms for more than a year [46], or with a complex case history [30].

Thirteen publications used conversation analysis, four used discourse analysis and one narrative analysis. Collections contained be- tween six and 51 interactions, and two publications were based on a single case analysis $[30,36]$. Between one and 18 healthcare providers participated, and the studies included between one and 116 patients, with 297 patients in total. The quality of the analysis (i.e. internal validity) was assessed as high for 11 studies, moderate for five and low for two studies. We refer to Appendix 3 for a general description of the data (i.e. external validity).

We could distinguish three main interrelated interactional and linguistic dimensions in the included studies: 1) symptom recognition; 2) double trouble potential; and 3) negotiation and persuasion. These dimensions were loosely related to the phases of the consultation, i.e. problem presentation, problem exploration, and diagnosis and treatment respectively.

\subsection{Symptom recognition}

Eight studies reported that recognition of symptoms as real and potentially severe is made relevant during the medical interaction. This is evident from additional interactional work addressing the legitimacy of the patient's visit and complaints. Below, we describe how patients pursue recognition and how healthcare providers legitimize patients' experience of unexplained symptoms.

\subsubsection{Patients in pursuit of recognition}

Patients claim legitimacy for their visit by presenting symptoms as worthy of medical attention. They describe experiencing abnormal [29] and involuntary symptoms [30] that threaten their daily functioning [31], despite having behaved as "morally sound" [29-31,39,47]. To underline their need for medical attention, patients refer to previous illnesses $[31,43]$ and inconclusive or negative results from previous examinations [29]. Patients thus position themselves as responsible patients suffering from symptoms that are not yet explained and in need of medical attention.
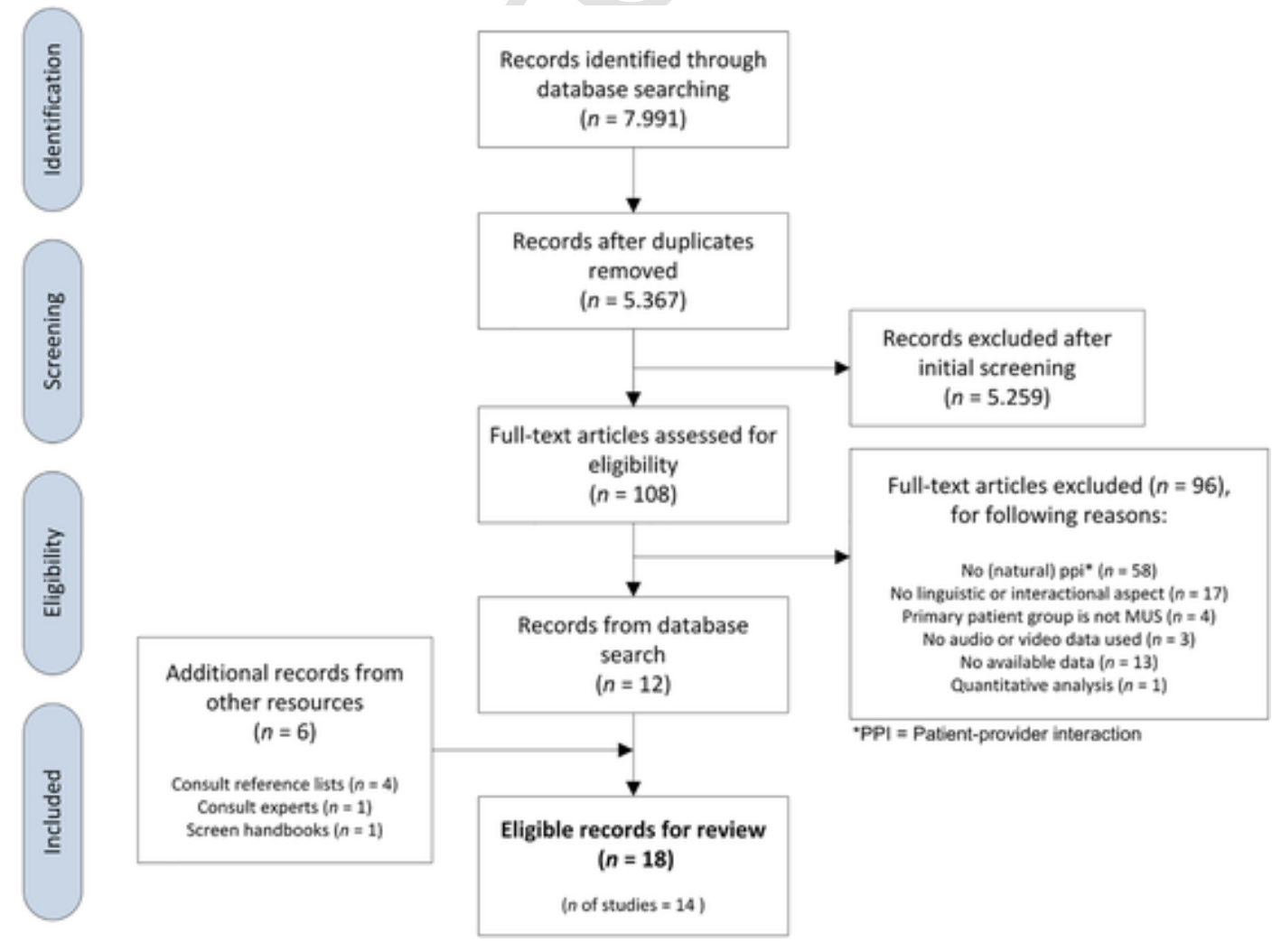

Fig. 1. Flow of inclusion. 
Table 2

Characteristics of included studies.

\begin{tabular}{|c|c|c|c|c|c|c|c|}
\hline Study & Year & Country & Setting & Illness & Sample & Analysis & Focus of analysis \\
\hline Aiarzaguena & 2013 & Spain & General practice & MUS & 10 & $\mathrm{CA}$ & Physicians' symptom explanations and patient responses \\
\hline Banks & 2001 & UK & CFS Clinic & CFS & 16 & DA & Lay and professional ideas about the nature of CFS \\
\hline Ekberg & 2015 & UK & Neurology & Seizures ${ }^{\mathrm{b}}$ & 38 & CA & History taking style and linguistic differential diagnostic pointers \\
\hline $\begin{array}{l}\text { Elderkin- } \\
\text { Thompson }\end{array}$ & 1998 & US & $\begin{array}{l}\text { Internal } \\
\text { medicine }\end{array}$ & MUS & 116 & NA & Narratives of somatising \& non-somatising patients \\
\hline Hyden & 1998 & Sweden & CFS clinic & CFS & 15 & DA & Negotiation of relevant symptoms, collaborative diagnosis work \\
\hline Monzoni $^{\mathrm{a}}$ & 2011a & UK & Neurology & FNS & 20 & $\mathrm{CA}$ & Passive vs. overt patient resistance \\
\hline Monzoni $^{\text {a }}$ & $2011 b$ & UK & Neurology & FNS & 20 & $\mathrm{CA}$ & Formulation effort and accounting activities \\
\hline Monzoni $^{\text {a }}$ & 2014 & UK & Neurology & FNS & 20 & $\mathrm{CA}$ & Psychosocial attributions \\
\hline Monzoni $^{\text {a }}$ & 2015 & UK & Neurology & FNS & 20 & CA & Self-restricted practices \\
\hline Monzoni $^{\text {a }}$ & 2016 & UK & Neurology & PNES & $17^{\mathrm{c}}$ & $\mathrm{CA}$ & Use of diagnostic labels and formulations \\
\hline Nessa & 1998 & Norway & General practice & IBS & 1 & DA & Relationship between observation and talk through medical gaze \\
\hline Opsommer & 2014 & $\begin{array}{l}\text { Switzer- } \\
\text { land }\end{array}$ & Physiotherapy & LBP & 6 & CA & $\begin{array}{l}\text { Exploration and assessment of pain experience during initial } \\
\text { encounters }\end{array}$ \\
\hline F. Roberts & 2014 & US & General practice & MUS & 21 & $\mathrm{CA}$ & Discussion of medication and addiction \\
\hline L. Roberts & 2018 & UK & Physiotherapy & LBP & 25 & $\mathrm{CA}^{\mathrm{d}}$ & Interruption and overlap by patients and providers \\
\hline Robson & 2016 & UK & Neurology & Seizures ${ }^{b}$ & 13 & $\mathrm{CA}$ & Companion behaviour to differentiate between epilepsy and PNES \\
\hline Tarber & 2013 & Denmark & General practice & $\begin{array}{l}\text { Chronic } \\
\text { pain }\end{array}$ & 1 & CA & Orientations to the purpose of the encounter \\
\hline Toerien & 2011 & UK & Neurology & Seizures & 13 & $\mathrm{CA}$ & Offering of choices in providing treatment options \\
\hline Undeland & 2008 & Norway & General practice & MUS & 2 & DA & Discourse contributing to unexplainedness \\
\hline
\end{tabular}

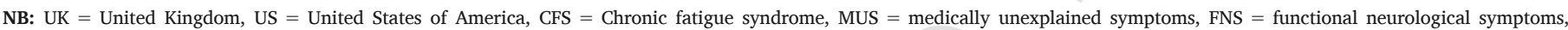
PNES = psychogenic non-epileptic seizures, IBS = irritable bowel syndrome, LBP = Low back pain, CA = conversation analysis, DA $=$ discourse analysis, NA = narrative analysis.

a Based on same dataset

b Compared PNES and epilepsy; this review only considered PNES consultations

c Focused on labelling of PNES, excluded patients with other FNS

d CA-based framework approach

Patients also account for the visit by illustrating the severity of their complaints with intensified language and variations in verb tenses. Intensified language includes extreme case formulations [48] such as "excruciating pain" [31], "very very sick" [46] or "terrible flushings" [30] (Box 1). Variations in verb tenses are also used to emphasize the severity of complaints. A patient in the study by Elderkin-Thompson, Silver and Waitzkin [31] uses non-progressive verbs (e.g. "I get numbness") to emphasize that the condition is static and unlikely to disappear in order to endorse the current need for medical attention, while another patient in F. Roberts \& Kramer's (2014) study uses progressive verbs (e.g. "it's getting, it's getting ba::d") to show the immediacy and discomfort of complaints. Another variation in verb use includes the use of the present tense when talking about past events (e.g. "the pain increases" rather than "the pain increased") [31].

After the diagnostic phase, patients still perform interactional work in pursuit of recognition. They comment on the diagnosis [29], present additional symptoms [29], express their concerns [30] or return to topics discussed earlier [30]. The data suggest that the purpose of this additional interactional work is to make sure all potentially relevant problems are evaluated.

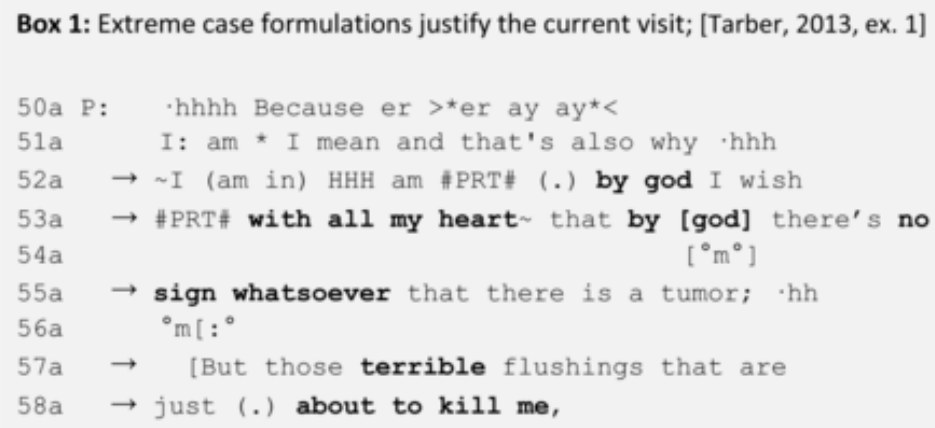

(NB: Extreme case formulations in bold [emphasis added])

The patient demonstrates her resistance to being sick with dramatizing expressions in lines 52a55a ("by god I wish with all my heart there's no sign whatsoever that there is a tumor"). Yet at the same time, she stresses the severity of her complaints with extreme case formulations ("terrible flushings that are just (.) about to kill me"). This choice of wordings legitimizes the claims that are made (42) and emphasizes that the complaints are rather severe and unlikely to disappear without any appropriate treatment.

Box 1. Extreme case formulations justify the current visit; [Tarber, 2013, ex. 1]. 


\subsubsection{Healthcare providers validating the experience of unexplained symptoms}

We could distinguish explicit and implicit approaches to support a patient's decision to seek medical care. Healthcare providers explicitly approve of the patient's story [47] or current visit (e.g. "it is good that you came since you have been having pain for a few days already" [36]) to legitimize symptom experiences. Implicit support includes providing diagnostic labels and syndrome descriptions that are in common use for MUS (e.g. "chronic fatigue syndrome") $[29,36]$. Such medical labels transform patients' subjective experience of symptoms into diseases that are generally recognized by society [29]. Another implicit approach to legitimize the experience of complaints is providing tangible explanations (see Box 2) that are co-constructed with patients [46]. This approach allows patients to relate explanations to their personal experiences.

It was found that if healthcare providers do not acknowledge patients' experience of symptoms, that arouses defensiveness and hopelessness in those patients [47]. A neutral stance towards patient's prior talk about illness behaviour leads to more elaborate accounts by patients [39], while neglecting the emotional content does not provide the acknowledgement that patients are pursuing [30].

\subsection{Double trouble potential}

Based on the evidence reported in 12 studies, we noted that consultations about MUS carry a double trouble potential that may hinder successful communication between healthcare providers and patients. The double trouble potential refers to two different aspects that we recognized in the included studies: pre-existing differing ideas about the origin of symptoms, and the differing knowledge domains of healthcare providers and patients. The latter is based on CA research about epis- temics [49], which Monzoni et al. [40,41] related to the context of MUS.

\subsubsection{Differing views on symptoms}

Healthcare providers and patients often have differing views about the nature of MUS. Some patients do not accept psychosocial explanations for their symptoms [31], as they provide accounts of physical causes, while healthcare providers provide explanations in which the patient's mental functioning causes the complaints [43]. Patients may also focus on the pervasive consequences of symptoms, whereas their healthcare providers are mapping out the onset and duration of complaints [30].

Patients exhibit resistance - passively or overtly - during consultations when these conflicting ideas become manifest. Passive resistance consists of remaining silent when turn-taking could take place $[42,46]$ and minimal continuers or acknowledgement tokens (e.g. "hm $\mathrm{hm}$ ") $[37,42]$. Overt resistance is performed with rejections or disagreements [e.g. "no:::", 41], questioning the explanation (e.g. "well I don't know, if it'll be tha:t") [31,46] or epistemic claims with extreme case formulations (e.g. "I never remember what happens during a seizure" [44]). Patients sometimes affirm potential psychosomatic attributions for symptoms in general, but they deny the possibility for their own case $[41,42,46]$.

Expression of passive or overt resistance mainly depends on two interactional features. First, sometimes healthcare providers do not invite patients to respond or leave no room for them to do so $[42,46]$. For instance, symptom explanations in extended turns cast patients as passive recipients of the diagnosis [37]. The second feature relates to the phase of the consultation. Patients mainly display passive resistance during the diagnostic phase and express their disagreement more overtly during discussion of psychosocial attributions or treatment recommendations [41] (Box 3).

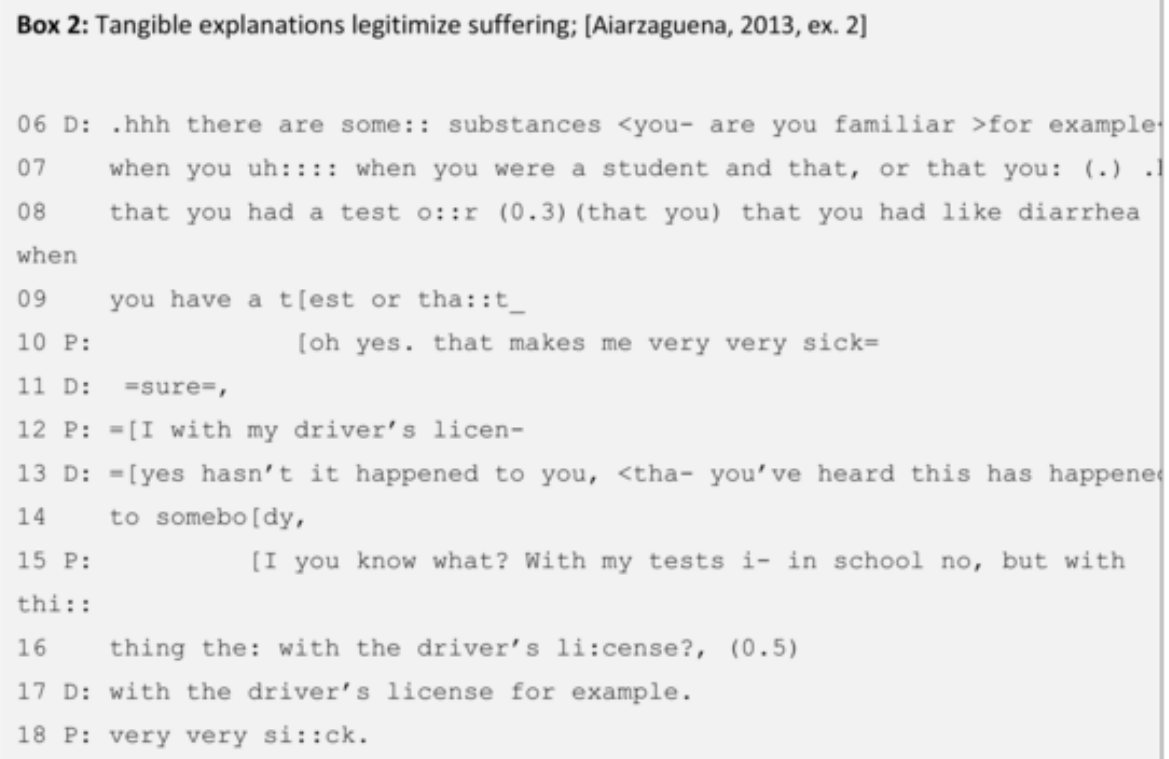

In lines 6 to 9 , the healthcare provider describes a situation that the patient might have encountered in her life, i.e. symptoms due to exam stress. The patient relates this explanation to her experience of getting sick during her driver's licence exam, thereby translating the provider's hypothetical example into a personal experience. Such elaborate responses in which patients show extensive agreement with the physician's explanation indicate that tangible explanations are "legitimizing and exculpating" (39).

Box 2. Tangible explanations legitimize suffering; [Aiarzaguena, 2013, ex. 2]. 


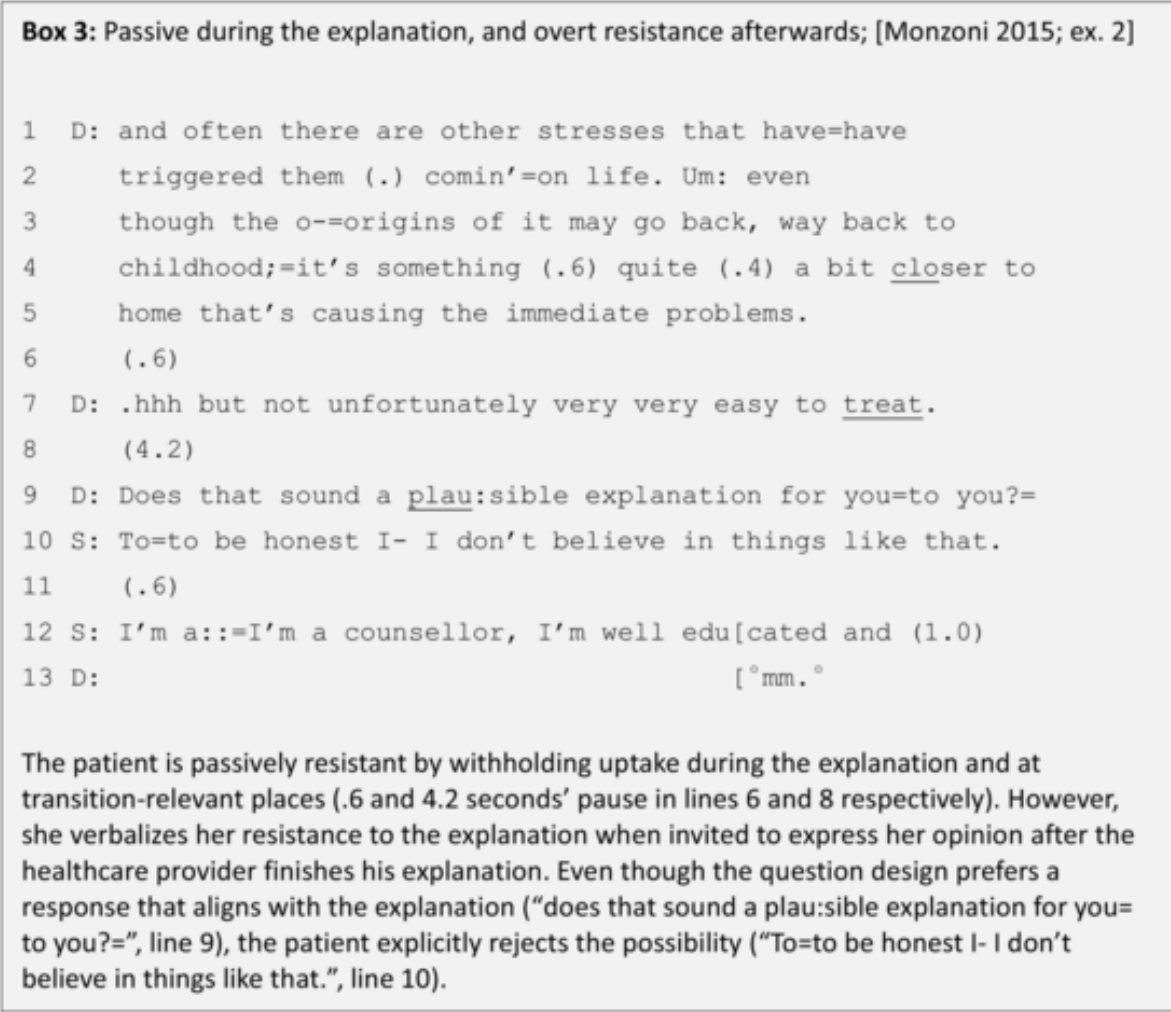

Box 3. Passive during the explanation, and overt resistance afterwards; [Monzoni 2015; ex. 2].

\subsubsection{Differing knowledge domains}

Another determinant for double trouble potential in consultations about MUS relates to the differing knowledge domains of healthcare providers and patients [cf. 40, 41]. When healthcare providers diagnose MUS, they cannot rely on their professional authority alone (the epistemics of expertise [49]), because their sources of information (e.g. test results) are inconclusive. Instead, healthcare providers rely on patient experiences, i.e. information that pertains to the patient's domain (the epistemics of experience [49]) [29,47]. Healthcare providers and patients orient to this knowledge asymmetry with different turn design features. For instance, patients do not overtly refute conclusions based on test results, whereas healthcare providers are cautious when raising psychosomatic attributions.

We could distil three practices that demonstrate how healthcare providers explain MUS with caution, i.e. 1) vagueness and mitigation, 2) detached footing, and 3) indirect constructions. First, psychosocial attributions are introduced with vague language (e.g. "things in your life or have been in your life") $[42,46]$ and mitigated constructions (e.g. "maybe it sounds a little strange" $[37,42,46]$. With such language use, healthcare providers demonstrate how they approach symptom explanations with considerable caution because they are in the patient's knowledge domain (Box 4).

Second, healthcare providers often do not attribute the explanation directly to the patient's current situation, but rather to what others in similar situations may experience (e.g. "may undermine (.8) > people's confidence $<$ ", Box 4, lines 9-10). Such detached footing [50] allows the healthcare provider to mention potentially difficult topics and gives room for patients to display disagreement (e.g. accepting an explanation, but denying it for their own case) $[37,39,42]$. Finally, symptom explanations are communicated cautiously with indirect linguistic constructions. These constructions include general non-medical labels (e.g. 'emotions' instead of 'psychological') [42] and negations (e.g. "it is not epilepsy") [37,38,40,41], which help to avoid using direct terms (e.g. 'dissociation').

\subsection{Negotiation and persuasion}

We discuss data from 17 studies that reported on the relationship between how healthcare providers gather information and how they provide acceptable explanations to patients. We label specific communicative actions of healthcare providers that pursue patient acceptance of symptom explanations or treatment recommendations as having a persuasive orientation [cf. 51].

\subsubsection{Information gathering}

Question-answer sequences are a central device during the history-taking phase [29]. Some consultations start with open-ended questions (e.g. "Tell me") [29,52] but opening and follow-up questions are frequently closed-ended and category-constrained (e.g. "So when did you fi:rst have these attacks") $[28,29,43,52]$. By doing this, healthcare providers control what information is relevant for them $[29,30]$ and when patients' answers suffice, e.g. by interrupting patients in the midst of their accounts $[28,53]$. Interruptions serve to seek or clarify information, which may enhance communication or it may lead to parallel conversations where each follows their own agenda [53].

If healthcare providers and patients disagree about the relevance of certain symptoms, traditional question-answer sequences may become problematic for patients as their response options are rather limited [29]. We observed several approaches patients use to resist healthcare providers' history-taking questions. Patients state their inability to answer a question with epistemic disclaimers (e.g. "I don't know"), turn to their companions, dispute the relevance of previous questions (e.g. "you'll have to answer that because") [44] or expand their answers to other issues they consider important $[30,31,44]$. A final approach includes reframing information, which encompasses the framing of their actions to refit certain diagnostic criteria (e.g. "I haven't been doing 


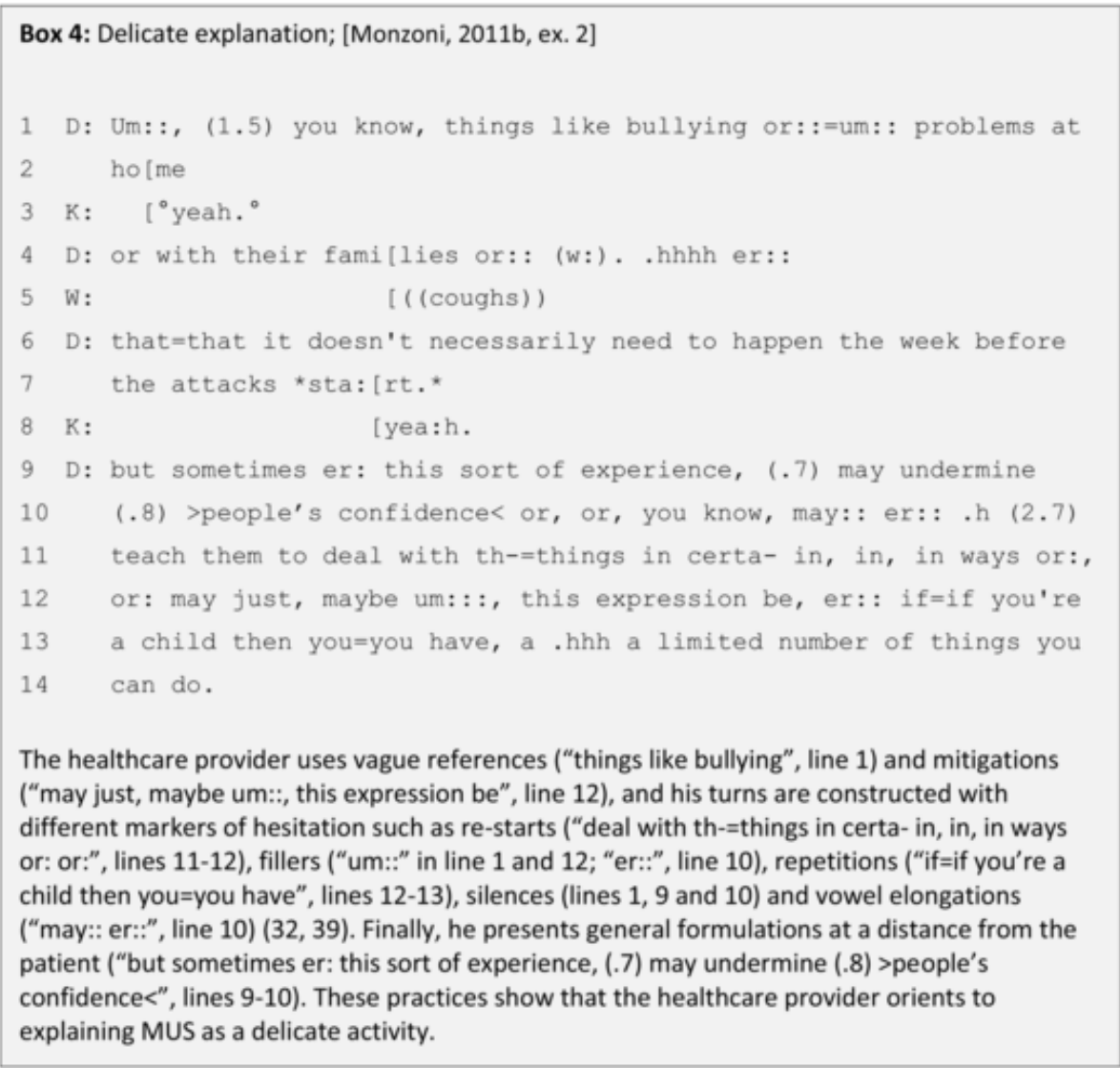

Box 4. Delicate explanation; [Monzoni, 2011b, ex. 2].

it [walking] since I've been like this, [...], but I'm usually out about an hour each day walking”) [29]. Healthcare providers' questioning thus heavily influences the possibility to obtain agreement about what is going on with patients, while patients implicitly influence the relevance of topics to accommodate their own agenda.

\subsubsection{Working towards agreement}

Healthcare providers pursue agreement with patients because disagreement about the diagnosis hinders further management [29,31,41]. They engage in (subtle) communicative actions that to establish acceptable explanations within the medical interaction [37]. We label such actions constituting persuasive conduct [51]. These actions may avoid friction and enhance agreement and could, eventually, lead to (psychological) treatment. Persuasive conduct occurs even when patients already aligned or extensively agreed with their healthcare provider $[40,46]$. We could distinguish three kinds of communicative actions in persuasive conduct, i.e. 1) tailoring explanations, 2) framing, and 3) subtle action recommendations.

First, healthcare providers tailor symptom explanations by referring to relatable situations [46] or issues discussed earlier [42]. They first solicit the patient's perspective before delivering the diagnosis $[37,42]$ and sometimes elicit patients' responses with questions such as "does that sound like a plausible explanation to you?" [37,42] or "How do you see it?" [46]. Though some patients disagree with such retrospective perspective display sequences, a co-construction of symptom explanations promotes agreement and validates the patient's knowledge of symptoms [47].

Second, healthcare providers elicit agreement with rhetorical devices that align with the patient's view. They frame explanations with plain, simple language in terms of physiology rather than psychology (e.g. "that's caused by the same chemical as gives rise to fatigue") [43]. Delicate issues that pertain to the patient's knowledge domain are re- placed with technical, medical terms (a "language of chemistry"), which allows healthcare providers to discuss psychosocial attributions without mentioning any (potentially stigmatizing) psychological diagnoses [43].

Thirdly, healthcare providers continue their persuasive conduct when making treatment recommendations $[30,40,45]$. Common treatment opportunities for MUS are limited to psychological or behavioural interventions [43], but patients often pursue reassurance [31] or somatic treatments $[41,43]$. When patients resist psychosocial explanations, they implicitly invalidate a diagnosis and thus the rationale for psychological treatment $[36,41]$. As a consequence, healthcare providers cautiously [40] recommend psychosocial treatment opportunities rather than other options. They introduce treatment options such as further testing or increasing drug intakes, while framing psychotherapy as preferred ("another reason why why you might want to choose this option") to other possibilities ("I am a bit reluctant to go with that option") [45]. This suggests that healthcare providers continue their persuasive conduct until the end of the consultation.

\section{Conclusions and discussion}

\subsection{Conclusions}

This review aimed to gain a detailed understanding of communication practices and their functions in consultations about MUS. By carefully analysing 18 studies of natural patient-provider interactions, we distinguished three main dimensions characteristic of MUS consultations. First, patients need their symptoms recognized during the medical interaction, as is demonstrated by their intensified language. Second, consultations have a double trouble potential due to the differing ideas and knowledge domains of healthcare providers and patients. This is manifested in patient resistance and cautious symptom explanations, e.g. with indirect linguistic constructions. Third, within the med- 
ical interaction healthcare providers construct symptom explanations that are acceptable for patients. Persuasive conduct such as tailoring explanations and framing facilitates consultation management by avoiding friction and eventually facilitating discussion of treatment options in these sometimes challenging interactions.

\subsection{Comparison with the literature}

A recent meta-synthesis of focus group and interview studies demonstrated that, according to doctors, patients and doctors have negative experiences in MUS interactions [1]. Patients risk legitimacy and want providers to acknowledge their symptom experience [54]. We show that this becomes manifest during the medical encounter. This is a universal issue for all patients presenting at the doctor's office [55], and can become problematic when symptoms have no medical explanation. Our review demonstrates that patients then account for seeking medical care [56], and claim legitimacy of the visit so their problems are treated as "doctorable" [55].

Previous research demonstrated that patients can have different explanatory models for the experience of complaints [1]. This review shows that both differing ideas and differing knowledge domains give potential for trouble in MUS consultations. This manifests itself in more complex turn designs (e.g. references to diagnostic evidence) that are generally observed for uncertain or disputed diagnoses [57-59]. This double trouble potential challenges the healthcare providers' role as a medical expert [59], which, we argue, could contribute to their feelings of discomfort and powerlessness [3].

Furthermore, Johansen and Risor [1] indicate that healthcare providers work towards successful consultation management with delicate approaches, e.g. by enhancing the relationship. This review indicates that they adopt several subtle strategies, such as tangible explanations or labelling symptoms, to help validate symptom experiences, though medical labels such as fibromyalgia may be perceived as an empty promise [60]. Healthcare providers carefully manoeuvre through the consultation to avoid predicating that patients suffer from a potentially stigmatized mental illness [61]. This avoids overt friction (too) early in the consultation.

We have demonstrated that instead of merely informing patients about a diagnosis and treatment options, healthcare providers engage in subtle (but sometimes extensive) persuasive interactional work in pursuit of patient acceptance. When healthcare providers use persuasion, it is not to sell patients something they might not want or need; rather, they take the sting out of a potentially difficult conversation. A negotiation of the patient's problem including the use of persuasive strategies thus serves to find common ground, which sometimes appears difficult to achieve [26].

Finally, the involvement of patients in the diagnostic phase is also observed in the delivery of bad news [62] and other situations for which cautious communication is warranted [63]. Specifically, the diagnostic news delivery is preceded by a (brief) pre-sequence that first elicits the patient's view. This enables healthcare providers to "confirm his [the patient's] experience, affirm the clinical diagnosis, and thereby co-implicate the patient's perspective in the delivery of the news" [64]. The diagnosis is thus communicated in a sensitive manner. 'Good' news indicating that no severe causes underlie the complaints is delivered as a form of bad news. Depending on patient expectations, 'good' diagnostic news is closely akin to bad news [65] without its potentially life-threatening consequences. Whereas some patients find the diagnosis of MUS a relief since they worried about potentially harmful underlying diseases, others perceive the diagnosis as 'bad' news since uncertainty remains about the cause (and treatment) of symptoms. Treating the delivery of a MUS diagnosis as a form of bad news may help facilitate the communication between healthcare providers and some patients with MUS during consultations that are frequently perceived as challenging.

\subsection{Strengths and weaknesses}

This review was the first to synthesize interactional studies of medical consultations about MUS. Rather than examining post-hoc patient views or provider experiences, we reviewed previous studies of naturally-occurring patient-provider interactions. We performed a systematic review according to PRISMA guidelines with extensive searches, independent selection and assessment of the included studies based on updated quality assessment instruments, and extensive discussions about the dimensions arising from the studies.

There are, however, some limitations to the review. First, consultations were mostly recorded in specialized healthcare settings with healthcare providers who had a special interest in the care of MUS patients. Patients experienced relatively severe complaints, and studies applied very diverse inclusion criteria since no gold standard exists for the operationalization of MUS (e.g. a minimum of four to six complaints [46], or follow-up consultations [41]). Though research has shown that certain practices in MUS consultations may be universal for different medical settings (i.e. neurology and psychotherapy) [66] or for different types of complaints [63], we found no evidence that specific practices described in individual studies account for all MUS interactions.

Second, though studies in this review $[28,44]$ and quantitative analyses $[35,67]$ suggest that healthcare providers and patients sometimes use language differently depending on the type of complaints patients present, our review did not compare consultations involving patients with medically unexplained symptoms with consultations where the symptoms did have a medical explanation. Future research is required to assess the generalizability of these findings for various medical settings and for different types of complaints.

Furthermore, we faced some challenges with the quality appraisal of the included studies. For instance, though carefully developed, our internal validity quality appraisal hardly discriminated between CA studies, whereas the remaining methods received weaker scores. It remains unclear whether this scoring difference is a result of a stronger internal validity for CA or a mere product of the quality assessment tool itself [68]. For this reason, we did not provide internal validity assessment tables for individual studies. Moreover, external validity was estimated through description of the data characteristics. Yet some studies did not provide data characteristics such as patients' demographic information. This informed us about the quality (or extent) of reporting rather than external validity issues. Future research should strive to further develop a method for appraising the internal and external validity of interactional and linguistic research, and critically assess its applicability for reviewing practices.

\subsection{Practice implications}

This systematic review shows that healthcare providers and patients manoeuvre carefully in medical consultations about MUS. Fine-grained analyses demonstrate that consultations carry a double trouble potential, and healthcare providers should treat the patient's visit as legitimate and attempt to overcome potential friction. Negotiation and persuasion may enhance agreement between healthcare providers and patients, which is required for the successful recommendation of (psychological) treatment. By eliciting patient views and tailoring symptom explanations, healthcare providers involve patients in constructing symptom explanations and treatment opportunities collaboratively. Attention to subtle linguistic and interactional aspects is key for the successful management of MUS consultations.

\section{Funding}

This study was supported by the Dutch Research Council NWO (grant number PGW.17.031). 


\section{Declaration of Competing Interest}

None.

\section{Acknowledgements}

The authors would like to thank Alice Tillema for her help in optimizing the database search, Niels Hazenberg and Ilona Plug for their help in selecting the studies, Victoria Land and Ruth Parry for providing the team with additional information related to their review, and Charlotte Albury for sharing their checklist for reporting CA studies.

\section{Appendix A. Supplementary data}

Supplementary data to this article can be found online at https://doi. org/10.1016/j.jpsychores.2020.109994.

\section{References}

[1] M L Johansen, M B Risor, What is the problem with medically unexplained symptoms for GPs? A meta-synthesis of qualitative studies, Patient Educ. Couns. 100 (4) (2017) 647-654.

[2] S Nettleton, 'I just want permission to be ill': towards a sociology of medically unexplained symptoms, Soc. Sci. Med. 62 (5) (2006) 1167-1178.

[3] L Wileman, C May, C A Chew-Graham, Medically unexplained symptoms and the problem of power in the primary care consultation: a qualitative study, Fam. Pract. 19 (2) (2002) 178-182.

[4] T C Olde Hartman, L J Hassink-Franke, P L Lucassen, K P van Spaendonck, C van Weel, Explanation and relations. How do general practitioners deal with patients with persistent medically unexplained symptoms: a focus group study, BMC Fam. Pract. 10 (2009) 68.

[5] T M Edwards, A Stern, D D Clarke, G Ivbijaro, L M Kasney, The treatment of patients with medically unexplained symptoms in primary care: a review of the literature, Ment Health Fam Med 7 (4) (2010) 209-221.

[6] A Aamland, K Malterud, E L Werner, Patients with persistent medically unexplained physical symptoms: a descriptive study from Norwegian general practice, BMC Fam. Pract. 15 (2014) 107.

[7] P Fink, M S Hansen, M L Oxhoj, The prevalence of somatoform disorders among internal medical inpatients, J. Psychosom. Res. 56 (4) (2004) 413-418.

[8] M Heijmans, T C Olde Hartman, E van Weel-Baumgarten, C Dowrick, P L Lucassen, C van Weel, Experts' opinions on the management of medically unexplained symptoms in primary care. A qualitative analysis of narrative reviews and scientific editorials, Fam. Pract. 28 (4) (2011) 444-455.

[9] V Teas-Gill, F Roberts, Conversation analysis in medicine, in: J Sidnell, T Stiver (Eds.), The Handbook of Conversation Analysis, Wiley-Blackwell, Malden, MA, 2013, pp. 575-592.

[10] J Heritage, J D Robinson, M N Elliott, M Beckett, M Wilkes, Reducing patients' unmet concerns in primary care: the difference one word can make, J. Gen. Intern. Med. 22 (10) (2007) 1429-1433.

[11] V Land, R Parry, J Seymour, Communication practices that encourage and constrain shared decision making in health-care encounters: systematic review of conversation analytic research, Health Expect. 20 (6) (2017) 1228-1247.

[12] R Parry, V Land, J Seymour, How to communicate with patients about future illness progression and end of life: a systematic review, BMJ Support. Palliat. Care 4 (4) (2014) 331-341.

[13] G W Noblit, R D Hare, Meta-Ethnography: Synthesizing Qualitative Studies, Sage, Newbury park, California, 1998.

[14] D Moher, A Liberati, J Tetzlaff, D G Altman, P. Group, Preferred reporting items for systematic reviews and meta-analyses: the PRISMA statement, PLoS Med. 6 (7) (2009) e1000097.

[15] T C Olde Hartman, M S Borghuis, P L Lucassen, F A van de Laar, A E Speckens, C van Weel, Medically unexplained symptoms, somatisation disorder and hypochondriasis: course and prognosis. A systematic review, J. Psychosom. Res. 66 (5) (2009) 363-377.

[16] R Hoedeman, A H Blankenstein, C M van der Feltz-Cornelis, B Krol, R Stewart, J W Groothoff, Consultation letters for medically unexplained physical symptoms in primary care, Cochrane Database Syst. Rev. 12 (2010) (CD006524).

[17] J A Swinkels, C M E van der Feltz-Cornelis, Multidisciplinaire richtlijn: Somatisch Onvoldoende verklaarde Lichamelijke Klachten en Somatoforme Stoornissen, Netherlands Institute of Mental Health Care and Addication and Dutch Institute of Health Care Improvement, Utrecht, 2010.

[18] C M van der Feltz-Cornelis, R Hoedeman, E J Keuter, J A Swinkels, Presentation of the multidisciplinary guideline medically unexplained physical symptoms (MUPS) and somatoform disorder in the Netherlands: disease management according to risk profiles, J. Psychosom. Res. 72 (2) (2012) 168-169.

[19] P Mistiaen, M van Osch, L van Vliet, J Howick, F L Bishop, Z Di Blasi, J Bensing, S van Dulmen, The effect of patient-practitioner communication on pain: a systematic review, Eur. J. Pain 20 (5) (2016) 675-688.

[20] R Parry, V Land, Systematically reviewing and synthesizing evidence from conversation analytic and related discursive research to inform healthcare communication practice and policy: an illustrated guide, BMC Med. Res. Methodol. 13 (2013) 69.

[21] D W Maynard, J Heritage, Conversation analysis, doctor-patient interaction and medical communication, Med. Educ. 39 (4) (2005) 428-435.

[22] P Drew, J Chatwin, S Collins, Conversation analysis: a method for research into interactions between patients and health-care professionals, Health Expect. 4 (1) (2001) 58-70.

[23] A W Frank, Just listening: narrative and deep illness, Fam. Syst. Health 16 (1998) 197-212.

[24] A Jaworski, N Coupland, The Discourse Reader, Routledge, Abingdon, Oxon, 2006.

[25] P Salmon, G M Humphris, A Ring, J C Davies, C F Dowrick, Primary care consultations about medically unexplained symptoms: patient presentations and doctor responses that influence the probability of somatic intervention, Psychosom. Med. 69 (6) (2007) 571-577.

[26] M den Boeft, D Huisman, L Morton, P Lucassen, J C van der Wouden, M J Westerman, H E van der Horst, C D Burton, Negotiating explanations: doctor-patient communication with patients with medically unexplained symptoms-a qualitative analysis, Fam. Pract. 34 (1) (2017) 107-113.

[27] A Ring, C F Dowrick, G M Humphris, J Davies, P Salmon, The somatising effect of clinical consultation: what patients and doctors say and do not say when patients present medically unexplained physical symptoms, Soc. Sci. Med. 61 (7) (2005) $1505-1515$.

[28] K Ekberg, M Reuber, Can conversation analytic findings help with differential diagnosis in routine seizure clinic interactions?, Commun. Med. 12 (1) (2015) $13-24$.

[29] L C Hyden, L Sachs, Suffering, hope and diagnosis: on the negotiation of chronic fatigue syndrome, Health 2 (1998) 175-193.

[30] C Tarber, Talking at Cross Purposes: Applying the Magnifying Glass to the Interaction between a Primary Care Practitioner and A "heartsink" Patient, Emotional Distress in the General Practice: A Conversation Analytic Study, Aarhus University, Aarhus, Denmark, 2013, pp. 118-145.

[31] V Elderkin-Thompson, R C Silver, H Waitzkin, Narratives of somatizing and non somatizing patients in a primary care setting, J. Health Psychol. 3 (3) (1998) 407-428.

[32] P Lucassen, R Reis, M Dees, W Assendelft, Kwalitatief onderzoek, in: M Offringa, P Assendelft, R Scholten (Eds.), Inleiding in Evidence-Based Medicine, Bohn Stafleu van Loghum, Houten, Netherlands, 2018, pp. 107-119.

[33] B C O'Brien, I B Harris, T J Beckman, D A Reed, D A Cook, Standards for reporting qualitative research: a synthesis of recommendations, Acad. Med. 89 (9) (2014) 1245-1251.

[34] C. Albury CHecklist for Conversation Analysis reporTing (CHAT)http://www. equator-network.org/library/reporting-guidelines-under-development/reporting guidelines-under-development-for-other-study-designs/\#912018

[35] I A Stortenbeker, J Houwen, P L B J Lucassen, H W Stappers, W J J Assendelft, S van Dulmen, T C Olde Hartman, E Das, Quantifying positive communication: Doctor's language and patient anxiety in primary care consultations, Patient Educ. Couns. 101 (9) (2018) 1577-1584.

[36] J Nessa, K Malterud, "feeling your large intestines a bit bound": clinical interaction--talk and gaze, Scand. J. Prim. Health Care 16 (4) (1998) 211-215.

[37] C M Monzoni, M Reuber, Linguistic and interactional restrictions in an outpatien clinic. The challenge of delivering the diagnosis and explaining the aetiology of functional neurological problems, in: F H G Chevalier, J Moore (Eds.), Producing and Managing Restricted Activities. Avoidance and Withholding in Institutional Interaction, John Benjamins, Amsterdam, 2015, pp. 239-270.

[38] C M Monzoni, M Reuber, Psychogenic Non-epileptic Seizures: How Doctors Use Medical Labels when they Communicate and Explain the Diagnosis, the Palgrave Handbook of Adult Mental Health: Discourse and Conversation Studies, Palgrave Macmillan, New York, NY, 2016, pp. 209-226.

[39] F Roberts, J S Kramer, Medication and morality: Analysis of medical visits to address chronic pain, in: H Hamilton, W S Chou (Eds.), Handbook of Language and Health Communication, Routledge, New York, NY, 2014, pp. 477-489.

[40] C M Monzoni, R Duncan, R Grunewald, M Reuber, How do neurologists discuss functional symptoms with their patients: a conversation analytic study, J. Psychosom. Res. 71 (6) (2011) 377-383.

[41] C M Monzoni, R Duncan, R Grunewald, M Reuber, Are there interactional reasons why doctors may find it hard to tell patients that their physical symptoms may have emotional causes? A conversation analytic study in neurology outpatients, Patient Educ. Couns. 85 (3) (2011) e189-e200.

[42] C M Monzoni, M Reuber, Neurologists' approaches to making psychosocial attributions in patients with functional neurological symptoms, in: E Graf, M Sator, T Spranz-Fogasy (Eds.), Discourses of Helping Professionals, John Benjamins, Amsterdam, the Netherlands, 2014, pp. 289-314.

[43] J Banks, L Prior, Doing things with illness - the micro politics of the CFS clinic, Soc. Sci. Med. 52 (1) (2001) 11-23.

[44] C Robson, P Drew, M Reuber, The role of companions in outpatient seizure clinic interactions: a pilot study, Epilepsy Behav. 60 (2016) 86-93.

[45] M Toerien, R Shaw, R Duncan, M Reuber, Offering patients choices: a pilot study of interactions in the seizure clinic, Epilepsy Behav. 20 (2) (2011) 312-320.

[46] J M Aiarzaguena, I Gaminde, I Clemente, E Garrido, Explaining medically unexplained symptoms: somatizing patients' responses in primary care, Patient Educ. Couns. 93 (1) (2013) 63-72.

[47] M Undeland, K Malterud, Diagnostic interaction: the patient as a source of knowledge?, Scand. J. Prim. Health Care 26 (4) (2008) 222-227.

[48] A Pomerantz, Extreme case formulations - a way of legitimizing claims, Hum. Stud. 9 (2-3) (1986) 219-229.

[49] J Heritage, Epistemics in conversation, in: J Sidnell, T Stivers (Eds.), Handbook of Conversation Analysis, Blackwell-Wiley, Sussex, 2013, pp. 370-394.

[50] V Teas-Gill, D W Maynard, On "Labelling" in actual interaction: delivering and receiving diagnoses of developmental disabilities, Soc. Probl. 42 (1995) 11-37 
[51] B Huma, E Stokoe, R O Sikveland, Persuasive conduct: alignment and resistance in prospecting "Cold" calls, J. Lang. Soc. Psychol. 38 (1) (2019) 33-60.

[52] E Opsommer, V Schoeb, 'Tell me about your troubles': description of patient-physiotherapist interaction during initial encounters, Physiother. Res. Int. 19 (4) (2014) 205-221.

[53] L Roberts, F Burrow, Interruption and rapport disruption: measuring the prevalence and nature of verbal interruptions during back pain consultations, J. Commun. Healthc. 11 (2) (2018) 95-105.

[54] A Werner, $\mathrm{K}$ Malterud, It is hard work behaving as a credible patient: encounters between women with chronic pain and their doctors, Soc. Sci. Med. 57 (8) (2003) 1409-1419.

[55] J Heritage, J Robinson, Accounting for the visit: Patients' reasons for seeking medical care, in: J C Heritage, D W Maynard (Eds.), Communication in Medical Care: Interactions between Primary Care Physicians and Patients, Cambridge University Press, Cambridge, 2006, pp. 48-85.

[56] C Heath, The delivery and reception of diagnosis in the general-practice consultation, in: P Drew, J C Heritage (Eds.), Talk at Work: Interaction in Institutional Settings, Cambridge University Press, New York, 1992, pp. 235-267.

[57] V T Gill, D W Maynard, On Labeling in actual interaction - delivering and receiving diagnoses of developmental-disabilities, Soc. Probl. 42 (1) (1995) 11-37.

[58] D W Maynard, On predicating a diagnosis as an attribute of a person, Discourse Stud. 6 (1) (2004) 53-76.

[59] A Peräkylä, Authority and accountability: the delivery of diagnosis in primary health care, Soc. Psychol. Q. 61 (4) (1998) 301-320.
[60] T Boulton, Nothing and everything: fibromyalgia as a diagnosis of exclusion and inclusion, Qual. Health Res. 29 (6) (2019) 809-819.

[61] L J Kirmayer, D Groleau, K J Looper, M D Dao, Explaining medically unexplained symptoms, Can. J. Psychiatr. 49 (10) (2004) 663-672.

[62] D W Maynard, The Perspective-Display Series and the Delivery and Receipt of Diagnostic News, Polity, Cambridge, 1991.

[63] R Parry, A video analysis of how physiotherapists communicate with patients about errors of performance: insights for practice and policy, Physiotherapy 91 (4) (2005) 204-214.

[64] D W Maynard, Delivering bad news in emergency care medicine, Acute Med. Surg. 4 (1) (2017) 3-11.

[65] D W Maynard, R M Frankel, On diagnostic rationality: Bad news, good news, and the symptom residue, in: J Heritage, D W Maynard (Eds.), Communication in Medical Care: Interaction between Primary Care Physicians and Patients, Cambridge University Press, Cambridge, 2006, pp. 248-278.

[66] C Burbaum, A M Stresing, K Fritzsche, P Auer, M Wirsching, G Lucius-Hoene, Medically unexplained symptoms as a threat to patients' identity? A conversation analysis of patients' reactions to psychosomatic attributions, Patient Educ. Couns. 79 (2) (2010) 207-217.

[67] M Reuber, C Monzoni, B Sharrack, L Plug, Using interactional and linguistic analysis to distinguish between epileptic and psychogenic nonepileptic seizures: a prospective, blinded multirater study, Epilepsy Behav. 16 (1) (2009) 139-144.

[68] M Dixon-Woods, R L Shaw, S Agarwal, J A Smith, The problem of appraising qualitative research, Qual. Saf Health Care 13 (3) (2004) 223-225. 